\title{
BMJ Open Comparing the accuracy of quantitative versus qualitative analyses of interim PET to prognosticate Hodgkin lymphoma: a systematic review protocol of diagnostic test accuracy
}

\author{
Vít Procházka, ${ }^{1}$ Miloslav Klugar, ${ }^{2,3}$ Veronika Bachanova, ${ }^{4}$ Jitka Klugarová, ${ }^{1,3}$ \\ Dagmar Tučková, ${ }^{2,3}$ Tomáš Papajík ${ }^{1}$
}

To cite: Procházka V, Klugar M, Bachanova V, et al. Comparing the accuracy of quantitative versus qualitative analyses of interim PET to prognosticate Hodgkin lymphoma: a systematic review protocol of diagnostic test accuracy. BMJ Open 2016;6:e11729.

doi:10.1136/bmjopen-2016011729

- Prepublication history and additional material is available. To view please visit the journal (http://dx.doi.org/ 10.1136/bmjopen-2016011729).

Received 29 February 2016 Revised 27 June 2016 Accepted 15 July 2016

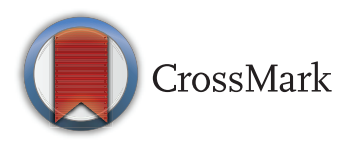

For numbered affiliations see end of article.

Correspondence to Dr Miloslav Klugar; miloslav.klugar@upol.cz

\section{ABSTRACT}

Introduction: Hodgkin lymphoma is an effectively treated malignancy, yet $20 \%$ of patients relapse or are refractory to front-line treatments with potentially fatal outcomes. Early detection of poor treatment responders is crucial for appropriate application of tailored treatment strategies. Tumour metabolic imaging of Hodgkin lymphoma using visual (qualitative) 18-fluorodeoxyglucose positron emission tomography (FDG-PET) is a gold standard for staging and final outcome assessment, but results gathered during the interim period are less accurate. Analysis of continuous metabolic-morphological data

(quantitative) FDG-PET may enhance the robustness of interim disease monitoring, and help to improve treatment decision-making processes. The objective of this review is to compare diagnostic test accuracy of quantitative versus qualitative interim FDG-PET in the prognostication of patients with Hodgkin lymphoma.

Methods: The literature on this topic will be reviewed in a 3-step strategy that follows methods described by the Joanna Briggs Institute (JBI). First, MEDLINE and EMBASE databases will be searched. Second, listed databases for published literature (MEDLINE,

Tripdatabase, Pedro, EMBASE, the Cochrane Central Register of Controlled Trials and WoS) and unpublished literature (Open Grey, Current Controlled Trials, MedNar, ClinicalTrials.gov, Cos Conference Papers Index and International Clinical Trials Registry Platform of the WHO) will be queried. Third, 2 independent reviewers will analyse titles, abstracts and full texts, and perform hand search of relevant studies, and then perform critical appraisal and data extraction from selected studies using the DATARI tool (JBI). If possible, a statistical metaanalysis will be performed on pooled sensitivity and specificity data gathered from the selected studies. Statistical heterogeneity will be assessed. Funnel plots, Begg's rank correlations and Egger's regression tests will be used to detect and/or correct publication bias.

Ethics and dissemination: The results will be disseminated by publishing in a peer-reviewed journal. Ethical assessment will not be needed; only existing sources of literature will be searched.

Trial registration number: CRD42016027953.

\section{INTRODUCTION}

\section{Background}

Classical Hodgkin lymphoma (cHL) is the most common lymphoid malignancy affecting patients below the age of 30. Incidence rates of cHL in the USA and Central Europe are comparable, with 2.7 new cases per 100000 men and women per year, and rates trending upwards. ${ }^{1}{ }^{2}$ Despite high cure rates and effective treatments for cHL, $20 \%$ of patients relapse or are refractory to front-line therapies. About $15 \%$ of these patients die within 5 years of diagnosis. ${ }^{3}$ Overall outcomes are unsatisfactory for patients with relapsed/refractory Hodgkin lymphoma (HL) who proceed to high-dose therapies and autologous stem cell transplants (SCTs). About $40-50 \%$ of SCT recipients relapse and require additional treatments. ${ }^{4}$ Given our entry into the era of novel 'targeted' drugs and immune modulators, identification of poor front-line treatment responders is a growing concern. ${ }^{5}$

Implementations of modern imaging methods such as positron emission tomography (PET)/CT have provided the capability to precisely assess tumour metabolic activity concurrent with an exact measurement of tumour burden. HL has been described as ubiquitously 18-fluorodeoxyglucose $\left({ }^{18} \mathrm{FDG}\right)$-avid. Revised response criteria for malignant lymphoma have therefore included tumour metabolic activity as a key parameter for determining remission status. Historically, complete metabolic responses have been assessed visually using either binary (positive/negative) or semiquantitative (Deauville) scales. ${ }^{6} 7$

FGG-PET is an inherently quantitative method that generates large amounts of metabolic and morphological data. Visual binary and semiquantitative PET analyses do not 
include quantitative and volumetric parameters (eg, total metabolic volume (TMV), total lesion glycolysis (TLG) or maximal standardised uptake volume (SUVmax)), and may be observer-biased. ${ }^{8}$ Recent studies have encouraged quantitative FDG-PET (QT PET) analyses to serve as novel biomarkers for staging and assessment of early (referred to as interim) and final malignant lymphoma tumour responses to treatments. ${ }^{9}{ }^{10}$ FDG-PET-based tumour metabolic activities at diagnoses were demonstrated to predict survival in HL and non-HL cases. Quantitative metabolic parameters have shown superiority when compared with semiquantitative assessments in untreated HL and primary diffuse large B-cell lymphoma cases. ${ }^{11-13}$ Given that personalised medicine has strongly emphasised individualised treatment approaches for all patients, evaluation of chemosensitivity is needed during oncology treatment. For cHL, those at risk of treatment failure may be identified by QT PET after a few cycles of therapy (referred to as 'interim PET').

Early (interim) visual assessment of cHL tumour metabolism has shown superiority when compared with standard prognostic scoring methods. ${ }^{14}$ Meta-analysis of these studies showed that interim FDG-PET had high prognostic value for identifying treatment failure. Unfortunately, interim PET has not been implemented in routine clinical practice due to the moderate quality of previous evidence and interstudy heterogeneity. ${ }^{15}$ Moreover, interim FDG-PET could not be used as a tool for tailored therapy as shown by results of two systematic reviews published by Sickinger et al. ${ }^{16}{ }^{17}$ One way to circumvent these barriers is to analyse QT PET results as a method of improving interim PET diagnostic accuracy and reproducibility. Several previous studies have investigated QT PET parameters during the interim period. For example, Rossi and colleagues demonstrated that interim PET after two cycles of anthracycline-based chemotherapy captured SUVmax ( $\triangle$ SUVmax) reductions as large as $71 \%$ below baseline. This technique identified positive responders with greater precision than did visual assessment alone. ${ }^{18}$ Quantitative $\Delta$ SUVmax achieved $85 \%$ diagnostic accuracy compared with just $76 \%$ from the visual method. Furthermore, positive predictive value increased by $24 \%$ (from $46 \%$ to $70 \%$ ) when the $\triangle$ SUVmax method was used in lieu of visual inspection. Additionally, Tseng and colleagues analysed 30 patients with cHL who were scanned at diagnosis and again during treatment. In this study, TMV, SUVmax and TLG were calculated together to determine cumulative changes during treatment regimens. Quantitative interim PET predicted both progressionfree and overall survival rates. ${ }^{19}$

To the best of our knowledge, a systematic review of the role of quantitative interim PET in patients with cHL has yet to be established. We hypothesise that measurements of quantitative tumour characteristics will improve diagnostic and predictive accuracy of interim PET. Thus, more successful candidates will be identified by interim PET for novel treatment approaches. The systematic review protocol described here has an extensive search strategy. It seeks to clarify the role of quantitative interim PET in cHL prognostication and influence practice by informing physician recommendations. Preliminary searches as of January 2016 were conducted using the MEDLINE, Prospero, JBI Library and Cochrane databases to establish whether previous systematic reviews on this topic were publicly available. No systematic reviews or guidelines related to this issue were discovered.

\section{Objective}

The objective of this review will be to compare diagnostic test accuracies between quantitative and qualitative interim PET methods with the aim of improving cHL prognostication.

\section{METHODS AND ANALYSIS \\ Methods}

This systematic review protocol was developed according to: (1) the Preferred Reporting Items for Systematic Reviews and Meta-Analysis Protocols (PRISMA-P),${ }^{20}$ and (2) the Joanna Briggs Institute (JBI) methodology for systematic reviews of diagnostic test accuracy. ${ }^{21}$ It has been enrolled with the PROSPERO prospective register of systematic reviews: CRD42016027953.

\section{Study eligibility}

Types of participants

The systematic review will consider all studies that investigated adult cHL (determined with the WHO diagnostic criteria), ${ }^{22}$ who were treated according to the current international guidelines. ${ }^{23}{ }^{24}$ Studies that included adolescents ( $\leq 18$ years old) will be excluded.

\section{Index test}

The systematic review will consider all studies that measure one or more of the following as an index test: QT PET, quantitative evaluation of interim FDG-PET by metabolic tumour volume, TLG or SUVmax. Only studies which used standardised international criteria for interim FDG-PET interpretation will be analysed. ${ }^{67}$

\section{Reference test}

The systematic review will consider studies that perform qualitative FDG-PET (QL PET) or visual evaluation of interim PET as a reference test.

\section{Diagnosis of interest}

The systematic review will consider studies that evaluate prognostic accuracy of QT PET in patients with cHL as calculated by changes in negative and positive predictive values when compared with QL PET.

\section{Types of studies}

The systematic review will only include diagnostic crosssectional study designs. 


\section{Search strategy}

A search strategy will be developed using medical subject headings (eg, MeSH for MEDLINE) and then adopted to query each database. Keywords related to the overarching topic will also be identified. The search strategy seeks to identify and include both published and unpublished work, and will therefore use a threestep search strategy. First, limited searches of MEDLINE and EMBASE will be undertaken, followed by analyses of keywords contained in the title, abstract and the index terms used to describe an article. Second, all identified keywords and index terms will be searched across all relevant databases. Third, reference lists from the newly identified reports and articles will be searched for additional studies. All studies with title and abstract in English will be considered for inclusion, regardless of the language used in the body of the manuscript. Studies published with no time restriction will also be considered for inclusion.

The databases to be searched include:

MedLine@Ovid, MEDLINE(R), Tripdatabase, Pedro, EMBASE, Cochrane Central Register of Controlled Trials, CINAHL and Web of Science.

Searches for unpublished studies will be performed using:

Open Grey, Current Controlled Trials, MedNar, ClinicalTrials.gov, Cos Conference Papers Index and International Clinical Trials Registry Platform of the WHO.

Example search strategy (MedLine@Ovid interface):

1. Hodgkin*;

2. Quantitative PET OR Metabolic Tumour Volume OR Total Tumour Glycolysis OR Standardized Uptake Value;

3. Qualitative PET OR Visual evaluation PET OR Visual analysis PET;

4. Diag* OR sensitivity OR specificity OR predictive;

5. 1 AND 2 AND 3 AND 4 .

\section{Study records}

Literature search results will be compiled and shared by the authorship team using EndNote V.X7, enabling collaborative study selection. Two reviewers (VP and JK) will independently screen and select studies for possible inclusion in two phases. First, titles and abstracts will be assessed. Second, all relevant full texts will be analysed. Any disagreements will be resolved by discussion and consultation of a third reviewer (MK), as necessary.

\section{Risk of bias in individual studies}

Papers selected for retrieval will be assessed by two independent reviewers (VP and DT) for methodological quality prior to inclusion in the systematic review. Assessments will use standardised critical appraisal instruments from the JBI Diagnostic Accuracy Test Assessment and Review Instrument (JBI-DATARI; QUADAS 2; see online supplementary appendix I). ${ }^{25}$
Any disagreements will be resolved by discussion and consultation of a third reviewer (MK), as necessary.

\section{Data collection process}

Data will be independently extracted by two reviewers (VP and MK) from studies included in the review using standardised data extraction tools from JBI-DATARI (see online supplementary appendix II).$^{25}$ Extracted data will include characteristics of the populations, index tests, reference tests and the diagnoses relevant to the systematic review objectives. Disagreements will be resolved during team discussions, as necessary.

\section{Data items/dealing with missing data}

Both generic and trade names of the index tests will be extracted. Diagnostic accuracy of index versus reference tests will be compared using sensitivity, specificity and receiver operating characteristics (ROC) readouts, as well as patient characteristics (eg, age, gender, given disease). Study authors will be contacted, as necessary, to provide relevant information for comparative assessments.

\section{Outcomes and prioritisation}

The primary outcome of this systematic review will be to compare diagnostic and prognostic accuracy of quantitative and qualitative PET results in patients with cHL.

We will seek data answering the following specific questions:

1. What was the rate of 5-year progression-free survival (PFS; followed from enrolment through the end of the study period)?

2. What is the predicted rate of treatment failure?

\section{Data synthesis}

All available diagnostic data will be pooled into a statistical meta-analysis using JBI-DATARI. Results from the included studies will be subjected to double data entry. Meta-analysis results will be presented with two graphical techniques. First, forest plots will illustrate sensitivity and specificity of each selected primary study by graphing the means and CIs. Means and CIs will also be in numeric form. Additionally, true-positive, false-positive, truenegative and false-negative values will be listed. Second, summary ROC curves will be created. The bivariate model for performing meta-analyses will be used.

\section{Assessment of heterogeneity}

Initially, clinical heterogeneity will be assessed by determining whether study inclusion criteria are sufficiently similar to the pooled results. If heterogeneity is found, characteristics of the differing studies will be carefully investigated. If it seems that heterogeneity is due to the existence of specific risks of bias in some studies, then the meta-analysis will be restricted to studies that do not contain those risks. To ensure sensitivity analysis, we will exclude all studies that are appraised as having a high risk of bias. 


\section{Subgroup analysis}

Subgroup analysis will be used for different age and gender characteristics. Another subgroup analysis will be used for cHL and different comorbidities according to their type and severity. Another subgroup analysis will be used for initial disease stage and type of chemotherapy given. If the data are available in primary studies, we will perform subgroup analysis according to: PFS; standardised PET using Body Phantom experiments.

\section{Metabias assessment}

To show potential reporting bias, we will use funnel plots if more than 10 studies are available. Begg's rank correlation and Egger's regression tests will be used for detecting and correcting publication bias.

\section{Confidence in cumulative evidence}

On the basis of the results and quality of evidence, the 'Grading of Recommendation Assessment, Development and Evaluation' (GRADE) tool will be used. ${ }^{26}$ Quality of evidence will be assessed across the domains of: risk of bias, consistency, directness, precision and publication bias. Quality will be assessed as: high (further research is very unlikely to alter confidence in the accuracy estimate), moderate (further research will most likely impact confidence in the accuracy estimate, and may change the estimate), low (further research is very likely to impact confidence in the accuracy estimate, and will most likely change the estimate) or very low (the accuracy estimate is very uncertain).

\section{ETHICS AND DISSEMINATION}

This systematic review protocol was crafted in February 2016. Next, the systematic review development team will begin performing the protocol described herein. Dissemination of results will be targeted at patients and oncology practitioners through publication in a peerreviewed journal. Ethical assessment is unnecessary as only existing sources of literature will be queried and evaluated.

\section{Author affiliations \\ ${ }^{1}$ Faculty of Medicine and Dentistry, Department of Hemato-Oncology, Palacký University in Olomouc, Olomouc, Czech Republic \\ ${ }^{2}$ Faculty of Medicine and Dentistry, Department of Social Medicine and Public Health, Palacký University in Olomouc, Olomouc, Czech Republic ${ }^{3}$ Faculty of Medicine and Dentistry, The Czech Republic (Middle European) Centre for Evidence-Based Health Care: An Affiliated Centre of the Joanna Briggs Institute, Palacký University in Olomouc, Olomouc, Czech Republic ${ }^{4}$ Division of Hematology, Oncology and Transplantation University of Minnesota, Minneapolis, Minnesota, USA}

Acknowledgements The authors would like to thank the Charlesworth Group for professional editing of this manuscript. Grant funding for this work was provided by the Faculty of Medicine and Dentistry, Palacký University in Olomouc, Czech Republic (IGA_LF_2016_001 and RVO: 61989592) and by Takeda Pharmaceuticals International AG (IISR-2015-101289).

Contributors VP and MK conceptualised and designed the study. All authors contributed to selection criteria development, risk of bias assessment strategy and data extraction. MK, JK and DT were methodologists. VP, VB and TP were Hodgkin lymphoma content experts. All authors (VP, MK, VB, TP) read, provided feedback and approved the final manuscript.
Funding Grant funding for this manuscript was provided by Faculty of Medicine and Dentistry, Palacký University in Olomouc, Czech Republic (IGA_LF_2016_001 and RV0: 61989592) and Takeda Pharmaceuticals International AG (IISR-2015-101289). The final review will disclose all financial and non-financial sources of support (ie, pharmaceutical companies); financial support will be drawn only from the publicly funded sources aforementioned.

Competing interests None declared.

Provenance and peer review Not commissioned; externally peer reviewed.

Data sharing statement The only data which the authors will miss could be missing data from primary studies. In this case, the authors will contact authors of primary research to ask for sharing the additional data with them.

Open Access This is an Open Access article distributed in accordance with the Creative Commons Attribution Non Commercial (CC BY-NC 4.0) license, which permits others to distribute, remix, adapt, build upon this work noncommercially, and license their derivative works on different terms, provided the original work is properly cited and the use is non-commercial. See: http:// creativecommons.org/licenses/by-nc/4.0/

\section{REFERENCES}

1. Shenoy $P$, Maggioncalda $A$, Malik N, et al. Incidence patterns and outcomes for Hodgkin lymphoma patients in the United States. Adv Hematology 2010;2011:725219.

2. Dusek L, Pavlík T, Májek O, et al. Estimating cancer incidence, prevalence, and the number of cancer patients treated with antitumo therapy in 2015 and 2020-analysis of the Czech National Cancer Registry. Klin Onkol 2015;28:30-43.

3. Canellos G, Rosenberg S, Friedberg J, et al. Treatment of Hodgkin lymphoma: a 50-year perspective. J Clin Oncol 2014;32:163-8.

4. Josting $A$, Rudolph $C$, Mapara $M$, et al. Cologne high-dose sequential chemotherapy in relapsed and refractory Hodgkin lymphoma: results of a large multicenter study of the German Hodgkin Lymphoma Study Group (GHSG). Ann Oncol 2005;16:116-23.

5. Moskowitz $\mathrm{CH}$, Matasar MJ, Zelenetz AD, et al. Normalization of pre-ASCT, FDG-PET imaging with second-line, non-cross-resistant, chemotherapy programs improves event-free survival in patients with Hodgkin lymphoma. Blood 2012;119:1665-70.

6. Barrington S, Mikhaeel G, Kostakoglu L, et al. Role of imaging in the staging and response assessment of lymphoma: consensus of the International Conference on Malignant Lymphomas Imaging Working Group. J Clin Oncol 2014;32:3048-58.

7. Meignan M, Barrington S, Itti E, et al. Report on the 4th International Workshop on Positron Emission Tomography in Lymphoma held in Menton, France, 3-5 October 2012. Leuk Lymphoma 2014;55:31-7.

8. Carlier T, Bailly C. State-of-the-art and recent advances in quantification for therapeutic follow-up in oncology using PET. Front Med (Lausanne) 2015;2:18.

9. Meignan M, Sasanelli M, Casasnovas R, et al. Metabolic tumour volumes measured at staging in lymphoma: methodological evaluation on phantom experiments and patients. Eur J Nucl Med Mol Imaging 2014;41:1113-22.

10. Meignan M. VI. FDG-PET as a biomarker in lymphoma: from qualitative to quantitative analysis. Hematol Oncol 2015;33:38-41.

11. Kobe C, Kuhnert G, Kahraman D, et al. Assessment of tumor size reduction improves outcome prediction of positron emission tomography/computed tomography after chemotherapy in advanced-stage Hodgkin lymphoma. J Clin Oncol 2014;32:1776-81.

12. Kanoun S, Rossi C, Berriolo-Riedinger A, et al. Baseline metabolic tumour volume is an independent prognostic factor in Hodgkin lymphoma. Eur J Nucl Med Mol Imaging 2014;41:1735-43.

13. Ceriani L, Martelli M, Zinzani PL, et al. Utility of baseline 18FDG-PET/CT functional parameters in defining prognosis of primary mediastinal (thymic) large B-cell lymphoma. Blood 2015;126:950-6.

14. Gallamini $A$, Hutchings $M$, Rigacci $L$, et al. Early interim 2-[18F] fluoro-2-deoxy-D-glucose positron emission tomography is prognostically superior to international prognostic score in advanced-stage Hodgkin's lymphoma: a report from a joint Italian-Danish study. J Clin Oncol 2007;25:3746-52.

15. Adams $H$, Nievelstein $R$, Kwee T. Prognostic value of interim FDG-PET in Hodgkin lymphoma: systematic review and meta-analysis. Br J Haematol 2015;170:356-66. 
16. Sickinger MT, von Tresckow B, Kobe $\mathrm{C}$, et al. Positron emission tomography-adapted therapy for first-line treatment in individuals with Hodgkin lymphoma. Cochrane Database Syst Rev 2015;1: CD010533.

17. Sickinger MT, von Tresckow B, Kobe C, et al. PET-adapted omission of radiotherapy in early stage Hodgkin lymphoma-a systematic review and meta-analysis. Crit Rev Oncol Hematol 2016;101:86-92.

18. Rossi C, Kanoun S, Berriolo-Riedinger A, et al. Interim 18F-FDG PET SUVmax reduction is superior to visual analysis in predicting outcome early in Hodgkin lymphoma patients. $J$ Nucl Med 2014;55:569-73.

19. Tseng D, Rachakonda L, Su Z, et al. Interim-treatment quantitative PET parameters predict progression and death among patients with Hodgkin's disease. Radiat Oncol 2012;7:5.

20. Moher D, Shamseer L, Clarke M, et al. Preferred reporting items for systematic review and meta-analysis protocols (PRISMA-P) 2015 statement. Syst Rev 2015;4:1.
21. Campbell J, Klugar M, Ding S, et al. The systematic review of studies of diagnostic test accuracy. In: JBI, ed. Joanna Briggs Institute reviewers' manual: 2015 edition/supplement. Adelaide, South Australia: The University of Adelaide, 2015:1-46.

22. Swerdlow S, Campo E, Harris N. WHO classification of tumours, volume 2; IARC WHO classification of tumours, No 2. Lyon, France: IARC Press, 2008.

23. Eichenauer DA, Engert A, Andre M, et alHodgkin's lymphoma: ESMO clinical practice guidelines for diagnosis, treatment and follow-up. Ann Oncol 2014;25(Suppl 3):iii70-5.

24. Hoppe RT, Advani RH, Ai WZ, et al. Hodgkin lymphoma, version 2.2012 featured updates to the NCCN guidelines. J Natl Compr Canc Netw 2012:10:589-97.

25. Campbell JM, Klugar M, Ding S, et al. Diagnostic test accuracy: methods for systematic review and meta-analysis. Int J Evid Based Healthc 2015;13:154-62.

26. Munn Z, Porritt K, Aromataris E, et al. Summary of findings tables for Joanna Briggs Institute systematic reviews. The University of Adelaide, South Australia: The Joanna Briggs Institute, 2014. 